\title{
BER Performance of IR-UWB Signal Based WBANs with BPPM
}

\author{
Ekhlas K. Hamza $^{\# 1}$, Ruaa S. Majeed ${ }^{* 2}$ \\ ${ }^{1,2}$ Control and Systems Engineering Department, University of Technology, 52 Street, Baghdad, Iraq \\ 1rekhlaskadhum@gmail.com \\ 2ruaasalam33@yahoo.com
}

\begin{abstract}
The basic requirement of Wireless Body Area Network (WBAN) is to send physiological signals acquired from implantable or wearable sensor nodes to a remote location. Low-power consumption is required for WBANs since most medical sensor nodes are battery operated. Impulse Radio-Ultra Wideband (IR-UWB) is a suitable wireless technology for the use in WBAN applications due to its inherent properties such as low power consumption,high data irate capability, low complexity hardware implementation, and small form factor.In this paper, the sensor nodes use two mechanisms to ensure that the transmit power is managed effectively. Firstly, the maximum allowable Full Bandwidth (FBW) transmit power is evaluated. Secondly, the number of Pulse Per Bit (PPB) can be dynamically changed according to the Bit Error Rate (BER) required value by using Binary Pulse Position Modulation (BPPM) scheme. These mechanisms enable the sensor nodes to operate at optimum power consumption and dynamic BER while maintaining a reliable data communication link.
\end{abstract}

Keywords - BER, BPPM, IR-UWB, WBAN.

\section{INTRODUCTION}

WBAN is a networking concept that has evolved with the idea of monitoring vital signals from low power and miniaturized in-body or on-body sensors. In a WBAN, data collected from the sensor nodes are transferred to a remotenodevia a wirelessmedium, where the data is forwarded to a higherlayer application to be interpreted [1].

The human body is a medium that poses numerous wireless transmission challenges. Unlike air, the body is composed of varied components that are not predictable and will change as the patient ages, gains or loses weight, or even changes in posture. Recent works suggested that for wireless communication inside the human body, the tissue medium acts as a channel through which the information is sent as electromagnetic (EM) radio frequency (RF). So in WBAN, information is transmitted as electromagnetic (EM) radio frequency (RF) waves [2].

Medical sensors involved in WBAN communicationare battery operated. Hence, they shouldconsume low power. This paper presents IR-UWB as a suitable wireless technology to achieve highdata rates while keeping power consumption and form factors small. Thus, the unique capabilities and potential applications of IR-UWB system have alreadydrawn a huge interestover the world.

This paper studies the effects of varying the number of PPB in the performance of IR-UWB system. Such effects are evaluated in terms of the BER for a BPPM scheme, which is the most popular form of modulation used for IR-UWB signal. The dynamic PPB scheme ensures that sensor nodes always transmit data with acceptable BER value.

The paper is organized as follows: Section IIstudiesan IR-UWB communication system. Section III discusses a simple BPPM technique which is used as the modulation scheme for the IR-UWB transmission. Section IV describes the implemented network in the simulation. Section V describes the transmission power optimization and BER analysis for multiple PPB and shows the results. Finally, section VI concludes the paper.

\section{OVERVIEW of IR-UWB SYSTEM}

IR-UWB is a novel wireless short-range technology. According to FederalCommunications Commission (FCC), IR-UWB communications operate in $0-960 \mathrm{MHz}$ and the $3.1-10.6 \mathrm{GHz}$ bands. IR-UWB signals have a fractional bandwidth largerthan 0.2 or at least $500 \mathrm{MHz}$. Since IR-UWB systems use ultra-wide bandwidth, the transmission rate of IR-UWB systems can go up to $20 \mathrm{Mbps}$. In the same time, the emission powerofIR-UWB must be kept below $-41.25 \mathrm{dBm} / \mathrm{MHz}$. As a result, IR-UWB devices canenjoy a muchlongeroperating time with a battery. On the other hand, low power transmission of signal limits the communication range (usually $0.1-2$ m) [3].

Outof the existingwirelessphysicallayer technologies, WirelessLocal Area Network (WLAN) standard is rarely used in WBAN applications because of its large power consumption. Zigbee, Bluetooth, and WLAN operate in the $2.4 \mathrm{GHz}$ unlicensed Industrial, Scientific and Medical (ISM) band; hence create interference issues to each other. Medical Implant Communication Services (MICS)band can only be used for low data rate WBAN applications due to its limited bandwidth capabilities. 
It can be concluded that the IR-UWB presents some unique benefits overotherwireless technologies in the designof WBAN sensornodesincluding the low power requirements, high data rate capability, small form factor, and uncomplicated circuit design. In terms ofinterference rejection, IR-UWB spectrumprovides a large bandwidth; hence, a sub-band ofIR-UWB can be selectedfor a particularapplicationsuchthat the interference fromother bands is minimized [4].

IR-UWB transmitter use simple short discrete pulses in order to transmit data. The IR-UWBpulse generation technique is shown in figure 1. IR-UWB pulses are generated by passing a squarewavesignaland its timedelayedversion through an XOR gate. Buffer with $3 \mathrm{v}$ supply voltage isused to introduce delay level to the signal.The data bit generated by the microcontroller is modulated by the IR-UWB pulse stream using a BPPM before entering the Low Pass Filter (LPF). Employing LPF in order to filterout the 0-1 GHz sectionof the IRUWB pulse spectrum. Thisportionof the spectrum is the highestpowercompared to restof the spectrum. Filtered spectrum is then shifted using a mixerand a Voltage ControlledOscillator (VCO) operating at $4 \mathrm{GHz}$. A band passfilter is used at the outputof the mixer in order to contain the IR-UWB signalswithin the $3.5-4.5 \mathrm{GHz}$ band.This technique offers the highestpower efficiency for anIR-UWB transmitter.An IR-UWB signalwith a bandwidthof $1 \mathrm{GHz}$ centered at $4 \mathrm{GHz}$, pulse width of $2 \mathrm{~ns}$ and Pulse Repetitive Frequency (PRF) of $100 \mathrm{MHz}$ is shown in figure 2.PRF affects the numberofspectrallinesandtheiramplitudesthat lie within a certain bandwidth. A higher PRF system tends to create a lessernumberofspectral lines that are higher in amplitude.

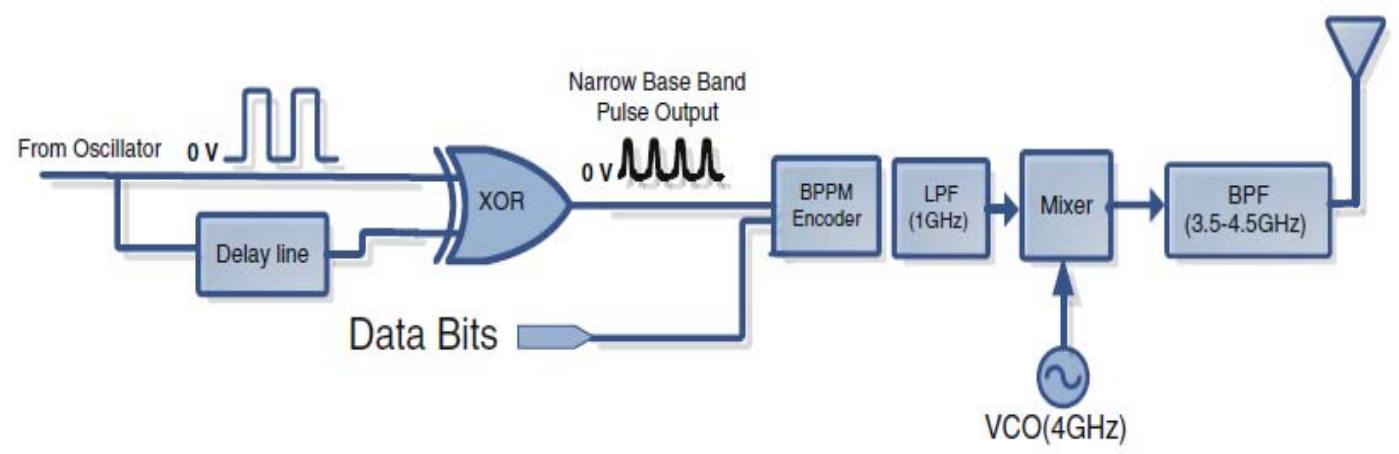

Fig. 1. IR-UWB pulse generation technique

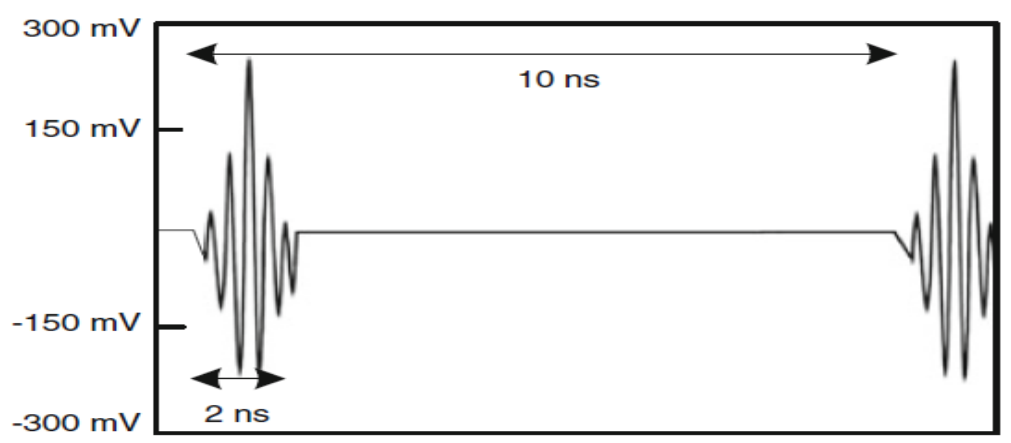

Fig. 2. IR-UWB pulse stream

\section{IR-UWB PULSE POSITION MODULATION SCHEME}

IR-UWB systems transmit short pulses to transmit data. Pulsed nature of IR-UWB transmitters enables the use of simplest modulation schemessuch as BPPM. BPPM scheme enables less complex hardware systems implementing IR-UWB communicationsystems and reduce the powerconsumption significantly.Also, it provides the best performance in terms of modulation efficiency and spectral performance. It is therefore more suitable forbatteryoperated WBAN applications.

BPPM scheme uses the positionof a pulse in two time slots to represent the value of an information bit, i.e. presence of a pulse in the first time slotindicates a ' 1 ' and that in the second time slotindicates a ' 0 '. In other words, a binary communication system can be established with a forward or backward shift of the pulse in time. When demodulating a BPPM signal, anIR-UWB receivercompares the energyof the receivedsignal in the twotime slots. If the energy in the first time slot is larger thanthat in the second time slot, a ' 1 ' is received; otherwise, a ' 0 ' is received. As shown in figure 3. Thus, the keyparameter in pulsepositionmodulation is the time delay ofeach pulse [5].Analytically, the signal can be represented as:

$\mathrm{S}_{\mathrm{i}}(\mathrm{t})=\mathrm{p}\left(\mathrm{t}-\tau_{\mathrm{i}}\right) \ldots$

Where $\mathrm{p}(\mathrm{t})$ is waveform at unmodulated nominal position, $\tau \mathrm{i}$ is time shift for ith modulation state [6]. 


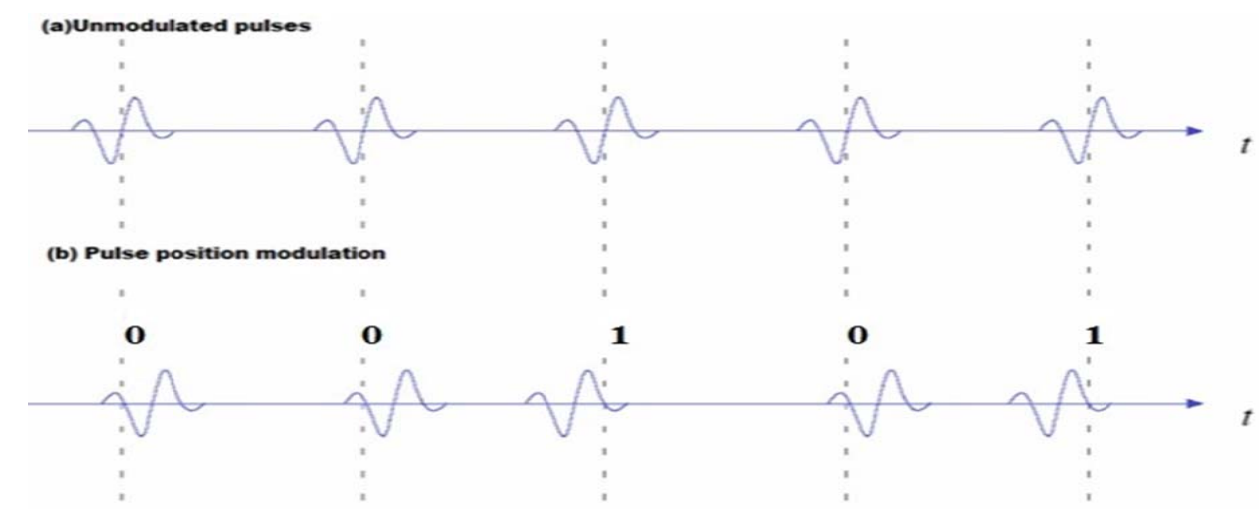

Fig. 3.(a) Unmodulated waves (b) BPPM modulated wave

SIMULATION MODELA WBAN used in the simulation is arranged such that the coordinator is placed at the center while the patientswith the sensornodesareplacedaround the coordinator preserving the Line-Of-Sight (LOS).

In this network, sensor nodes directly communicate with the coordinator using IR-UWB communication. Coordinator node acts as the centralcontrolling device of the proposed WBAN system. It is responsible for organizing and controlling the communication with multiple sensor nodes while maintaining an acceptable level of BER.

All the nodes are contained in a $10 \mathrm{~m} * 10 \mathrm{~m}$ (average hospital room area) MATLAB simulation environment. Each sensor node is placed at an averagedistanceof $2 \mathrm{~m}$ from the coordinator node. Considering a realistichospital scenario, a maximum of five patientsareassumed to enter the room during the simulation time. It should be noted that the BER increases significantly as the number of sensor nodes increases. In order to maintain an acceptable BER value, the maximum number of sensor nodes has to be limited to four for a single patient.Figure4 depicts the simulated network topology.

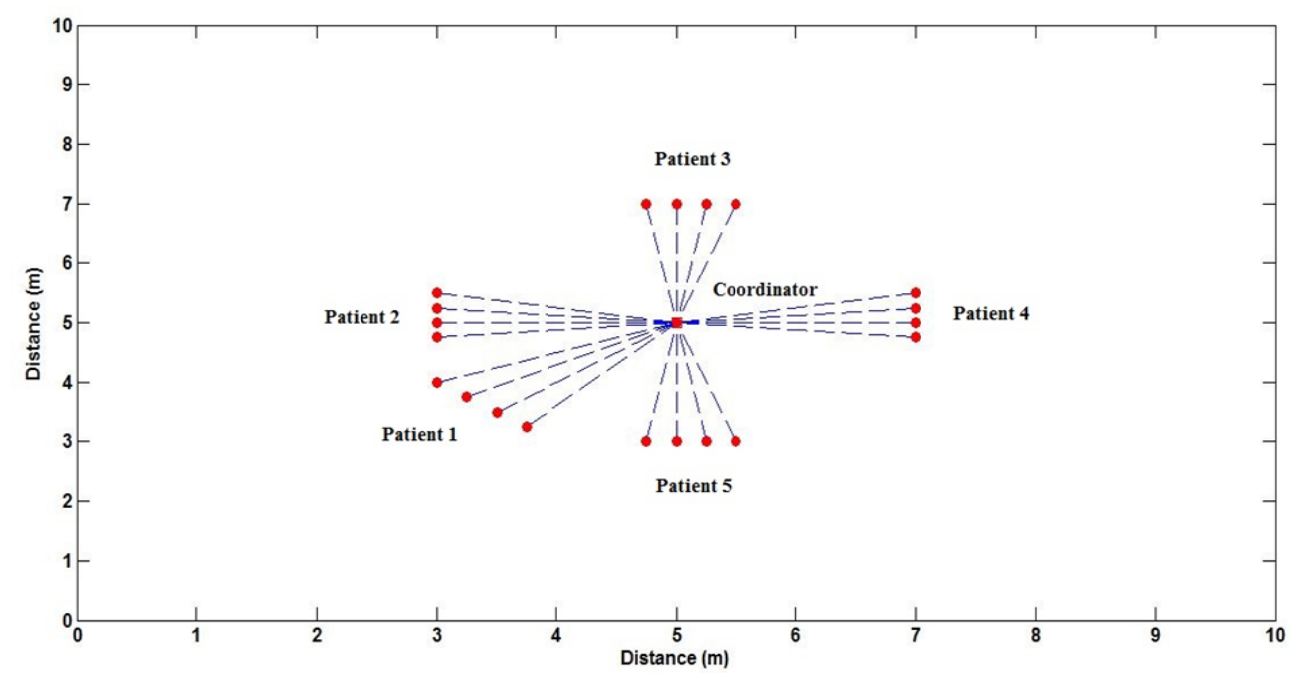

Fig. 4. Network topology

\section{TRANSMISSION POWER OPTIMIZATION}

The transmit power consumption ofsensor nodes used in the simulation is determinedby two limiting factors. Firstly, the maximum allowable FBW transmit power, whichdepends on the duty cycle of the pulse transmission, determines the maximum limitof the energyper UWB pulse. Secondly, the numberof PPB value determines the numberof UWB pulses sentwithin the bit transmission slot, which determines the energy consumption with in the transmission slot. However, these two factors are discussed in detailinthis section. Figure 5 depicts the use of 2 and 3 PPB schemesfor sending data bits. 


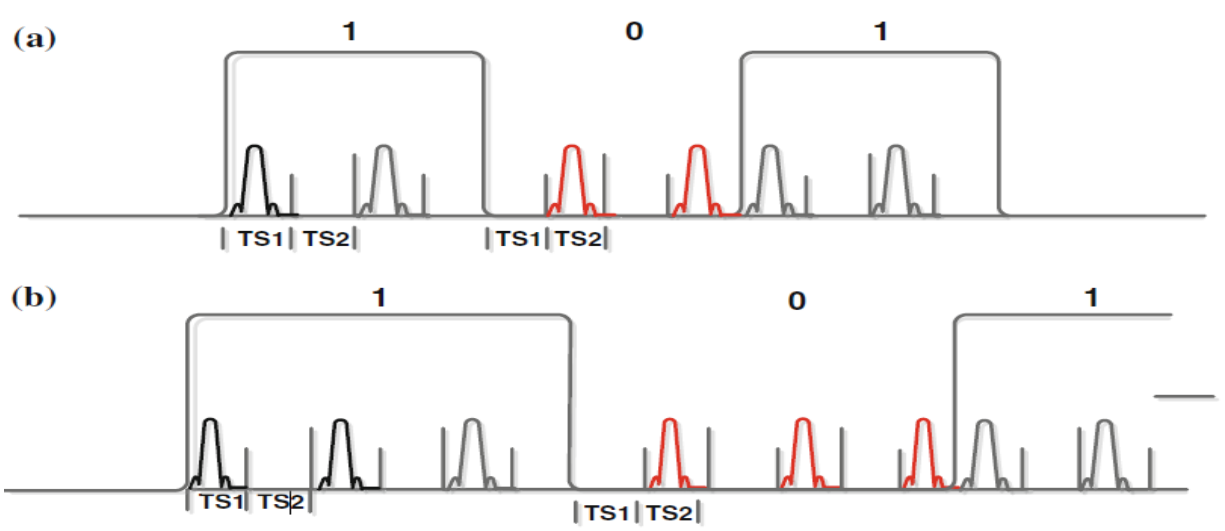

Fig. 5. Pulse train for ' 1 ' ' 0 ' ' 1 ' bit pattern using (a) Two PPB and (b) Three PPB in BPPM

\section{A. Transmission PowerLimitations}

According to the FCC regulations, an IR-UWB signal is a powerlimited by measuredFullBandwidth (FBW) peakpowerof $0 \mathrm{dBm}(1 \mathrm{~mW})$ andmeasuredaveragepowerdensityof $-41.25 \mathrm{dBm} / \mathrm{MHz}(75 \mathrm{nW} / \mathrm{MHz})$ [7]. The measurementof the average and peak power can be calculated easily using a spectrum analyzer in practice. For the average power measurement, the resolution bandwidth is $1 \mathrm{MHz}$ with an integration time of $1 \mathrm{~ms}$. A resolution bandwidth of between 1 and $50 \mathrm{MHz}$ can be used for the measurement of the peak power. The peak limit is dependent on the resolution bandwidth and varies according to [8]:

Peak power $=20 \log \left(\frac{\text { resolution bandwidth }}{50}\right) \quad \mathrm{dBm} \quad \ldots$

These measuredpower limitations can be converted to maximum allowable FBW transmitpower limits using (3) [9]:

Ppeak $\leq 7.5 \times 10^{-8}\left(\frac{B p}{R}\right)^{2} \times \frac{1}{\delta} \quad W \ldots$

Where Ppeak is the actual maximum transmitpowerof the IR-UWB signal, $B p=1 / \tau, \tau$ is the pulse width, $R$ is the $\mathrm{PRF}$ and $\delta$ is the duty cycle of pulse transmissioni slot based on a measurementdurationof $1 \mathrm{~ms}$. For the simulated system, a pulsewidthof $2 \mathrm{~ns}$ is used. Hence Bpin (3) is equal to $0.5 \mathrm{GHz}$ for the IR-UWB signals used in the simulations. Figure 6 shows the variation of maximum allowable FBW transmitpowervalueswith the duty cycle $(\delta)$ for a sensor node that generatesIR-UWB signalswith a PRF of $100 \mathrm{MHz}$ and a pulsewidthof $2 \mathrm{~ns}$. According to figure 6, the duty cycle of the IR-UWB signal should be kept within $10 \%$ in order to transmit at a maximum allowable power of $0.0186 \mathrm{~mW}(-17.3 \mathrm{dBm})$, which complies with the FCC limitations.

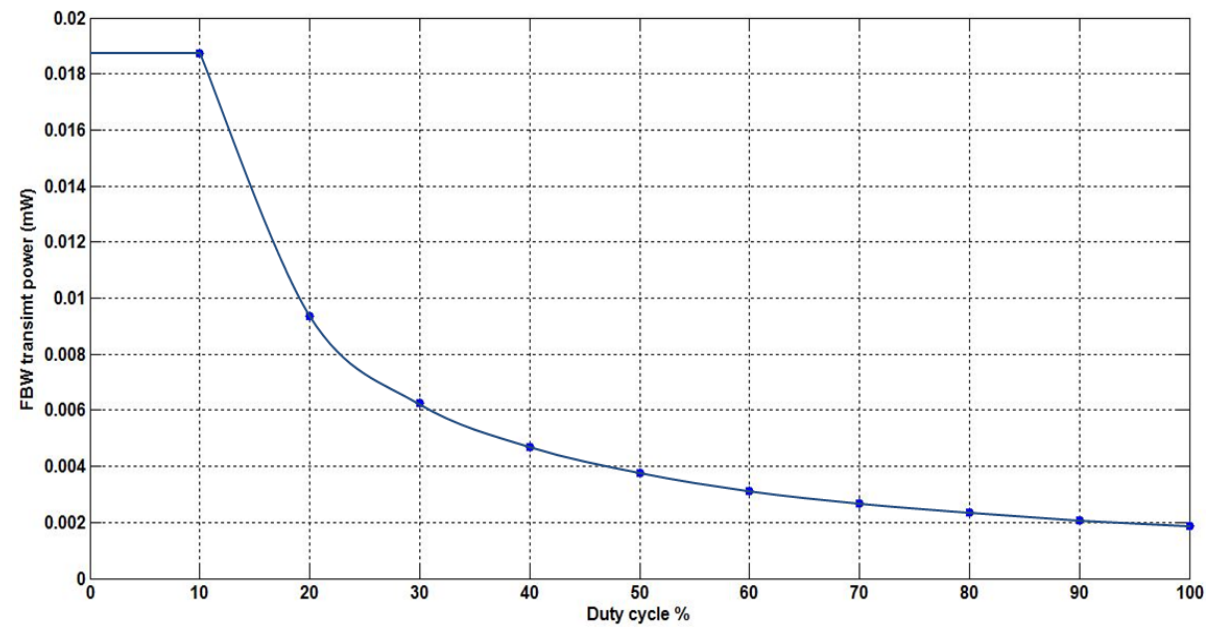

Fig. 6. Variation of FBW peakpowerwith duty cycle

\section{B. BER Analysis of Multiple PPB Scheme}

BER stands for bit error rate. It is the numberofbiterrorsdivided by the total numberof bits itransmitted during a studied time interval. It is usually used as a performancemeasurement in digital communications. The bit errors in a WBAN environmentmainlyoccur due to multipath interference and random fading of the IR-UWB signal that originates from reflection from various surfaces anddifferentabsorption characteristics of objects, such as various body surfaces and indoorequipment [10]. 
Since the powerrequired to transmit a databit is equal to the summationof the powerof a numberofpulsessent to represent that data bit, a considerablepowersaving can be achieved if the allocationof the numberof PPB can be dynamically changedaccording to the BER required value at the receiver end.

Assume that two identical sets ofdata are transmitted using the same transmitpower and same separation distance in a realistic WBAN environment that is susceptible to multipath interference and random fading with onedata set itransmitted using a higher PPB valueand the otherwith a lower PPB value. Thetransmit signal withhigher PPB transmissioniresults in a lower BER than a lower PPB transmissionifor the same separation distance in a realistic environment with fading and multipath interference.

Probability of error for single pulse detection of the receiver with BPPM modulationscheme can be derived from[11]:

$$
\mathrm{Pe}=\mathrm{Q}\left(\sqrt{\frac{(\mathrm{Ep} / \mathrm{No})^{2}}{2(\mathrm{Ep} / \mathrm{No}+\mathrm{Ts} \mathrm{B})}}\right) \cdots
$$

Where Pe is theprobabilityof error, $\mathrm{B}$ is the signal bandwidthof $1 \mathrm{GHz}$, Ts is the integrationperiodwhich is equal to the pulsewidthof $2 \mathrm{~ns}$ forthe simulation, Ep is the received signal energyduring the $2 \mathrm{~ns}$ integrationperiod and $\mathrm{Q}$ represents the $\mathrm{Q}$ function.

When multiple PPB is sent, it is assumedthat a bit is erroneous when more than halfthe pulses sent per that bit are erroneous. If $\mathrm{N}$ pulses are sent per bit, probability that a bitbeingerroneous can be obtainedby:

Pebit $=1-\sum_{\mathrm{i}=1}^{\left\lfloor\frac{N}{2}\right\rfloor}\left(\begin{array}{c}\mathrm{N} \\ \mathrm{i}\end{array}\right) \mathrm{p}^{\mathrm{i}}(1-\mathrm{p})^{\mathrm{N}-\mathrm{i}} \ldots$

Where $\mathrm{p}=\mathrm{Pe},\left(\begin{array}{c}\mathrm{N} \\ \mathrm{i}\end{array}\right)=\frac{\mathrm{N} !}{\mathrm{i} !(\mathrm{N}-\mathrm{i}) !}$ and $\left[\frac{N}{2} \mid\right.$ is the inferior integerpart of $\frac{\mathrm{N}}{2}$. Modulationcurvesshowing BER fordifferent number of PPB are obtained based on (5) and presented in figure7. It should be noted that the BER is plotted against pulse $\mathrm{Ep} / \mathrm{N} 0$ in this figure. Bitenergycan be obtained by the summationofpulseenergieshatrepresent the bit. Theresults in figure7showthat for the same Ep/N0, sendingmorenumberof PPB results in lower BER.

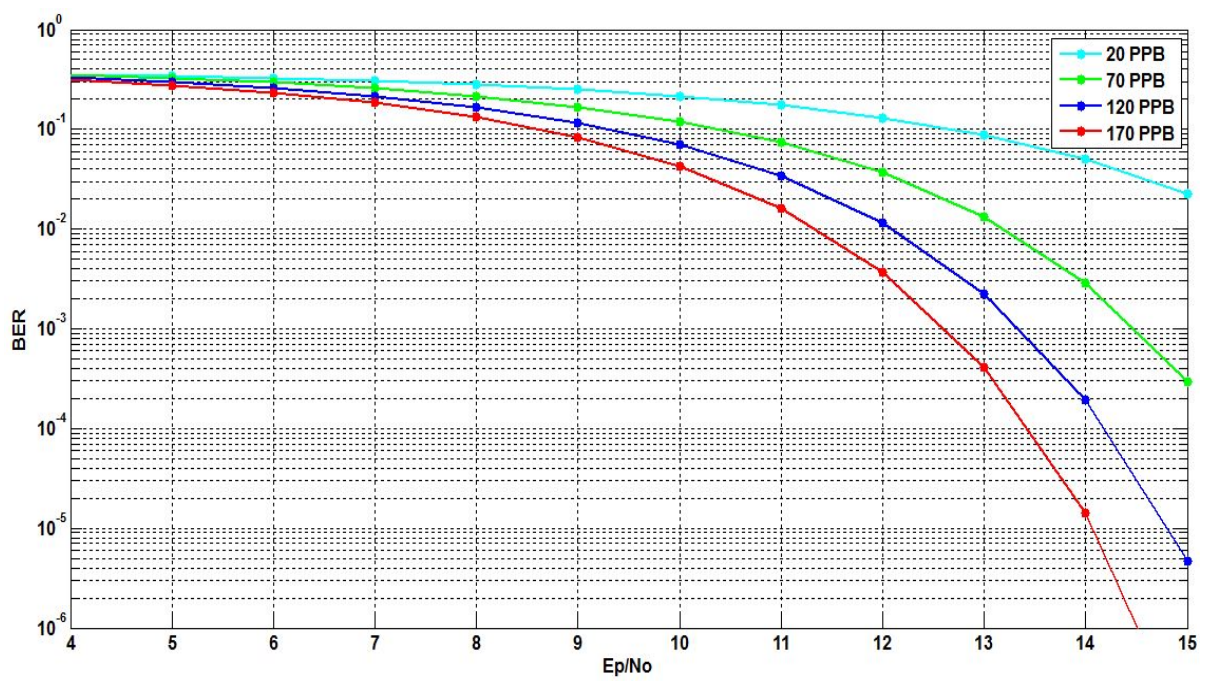

Fig. 7.BER versus pulse Ep/No (dB) curvesfordifferentnumberof PPB

From the above result, we can conclude that the numberof PPB can be dynamicallychanged in order to control the BER value. For best system performance, the optimum BER value is $10^{-4}$ for all sensor nodes, because a good throughput can be obtained with this value.

\section{CONCLUSION}

In this paper, the performance of IR-UWB system based WBAN was studied. A simple BPPM technique is used as the modulationscheme for the IR-UWB transmission. The FBW transmit power and PPB mechanisms are analyzed. Thesemechanisms lead to dynamic BER andpowercontrol at the sensor nodes, which helps to improve the reliability of communicationandpowerefficiencyofsensor nodes underdynamicchannel conditions.It can be concluded that the duty cycle of the IR-UWB signal should be kept within $10 \%$ in order to transmit at a maximum allowable power of $0.0186 \mathrm{~mW}$. Furthermore, when sending more number of PPB results in lower BER. 


\section{REFERENCES}

[1] M.R. Yuce, T.N. Dissanayake, et al."Wideband technology for medical detection and monitoring, recent advances in biomedicalengineering".Ed. by G.R Naik, ISBN: 978-953-307-004-9, InTech. 2009.

[2] Shahriyar, M. F. Bari, et al. "Intelligent mobile health monitoring system (IMHMS)". International Conference on ElectronicHealthcare, Springer. 2009.

[3] Broustis, S. V. Krishnamurthy, et al. "Multiband media access control in impulse-based UWB ad hoc networks". IEEE transactionsonmobile computing 6(4). 2007.

[4] H. Hongwei, X. Youzhi, et al. "Coexistence issues of $2.4 \mathrm{ghz}$ sensor networks with other rf devices at home".International Conference on Sensor Technologies and Applications, pp. 200-205. 2009.

[5] X. Cao, G. Huang, et al."Indoor UWB Communication System". Electrical and Computer Engineering. 2014.

[6] Banstola, R. Bera, et al. "Review and Design of UWB Transmitter and Receiver". International Journal of Computer Applications69(13). 2013.

[7] FCC 02-48 (First Report and Order), 2002.

[8] Chee Keong, Terence SP See, et al. "An ultra-wideband wireless body area network: evaluation in static anddynamic channel conditions". Sensors and Actuators A: Physical 180: 137-147. 2012.

[9] R.J. Fontana, E.A. Richley."Observations on low data rate, short pulse UWB systems". IEEE international conference on ultrawideband,pp. 334-338, 2007.

[10] Chen, Lingxiu. "An enhanced pulse position modulation (PPM) in ultra-wideband (UWB) systems." M.Sc. thesis, University of Northern Iowa, United States, 2014.

[11] Dubouloz, B. Denis, et al. "Performance analysis of LDR UWB non-coherent receivers in multipath environments". Ultra-Wideband, 2005. ICU 2005. 2005 IEEE International Conference on, IEEE. 2005. 